\title{
In vitro and in vivo antitumor effects of the recombinant immunotoxin IL6(T23)-PE38KDEL in multiple myeloma
}

\author{
DE-JUN GUO ${ }^{1-3}$, JIA-SHAN HAN ${ }^{2}$, YAN-SONG LI ${ }^{3}$, ZENG-SHAN LIU ${ }^{3}$, SHI-YING LU ${ }^{3}$ and HONG-LIN REN ${ }^{3}$ \\ ${ }^{1}$ College of Animal Science and Veterinary Medicine, Jilin University, Changchun, Jilin 130062; \\ ${ }^{2}$ College of Food, Heilongjiang Bayi Agricultural University, Daqing, Heilongjiang 163319; \\ ${ }^{3}$ Key Laboratory of Zoonosis Research, Ministry of Education, Institute of Zoonosis, \\ Jilin University, Changchun, Jilin 130062, P.R. China
}

Received February 23, 2012; Accepted May 23, 2012

DOI: $10.3892 / \mathrm{ol} .2012 .733$

\begin{abstract}
IL6(T23)-PE38KDEL is a chimeric molecule composed of interleukin 6 (IL6), missing the N-terminal 23 amino acids, and fused to a truncated mutant form of Pseudomonas exotoxin (PE38KDEL). The aim of this study was to evaluate this recombinant immunotoxin in terms of its specific cytotoxicity to IL6R-overexpressing multiple myeloma (MM) cells in vitro, as well as its antitumor effects and side effects in vivo. IL6(T23)-PE38KDEL was expressed in Escherichia coli, refolded and purified from inclusion bodies. The purified IL6(T23)-PE38KDEL was found to be selectively cytotoxic to IL6 receptor-positive tumor cells in vitro. $\mathrm{IC}_{50}$ values of IL6(T23)-PE38KDEL were evaluated by MTS assay. Toxicity and maximum-tolerated dose of IL6(T23)-PE38KDEL were determined in mice. The antitumor activity of IL6(T23)-PE38KDEL was evaluated in mice with MM through intravenous injection and interventional therapy. Intravenous administration of IL6(T23)-PE38KDEL caused a significantly increased survival time in treated mice, and exhibited dose- and time-dependent antitumor effects against MM mice. Moreover, complete tumor regression was
\end{abstract}

Correspondence to: Professor Zeng-Shan Liu, Key Laboratory of Zoonosis Research, Ministry of Education, Institute of Zoonosis, Jilin University, Changchun, Jilin 130062, P.R. China

E-mail: zsliu1959@126.com

Abbreviations: RIT, recombinant immunotoxin; PE, Pseudomonas exotoxin; IL6, human interleukin-6; IL6R, interleukin-6 receptor; sIL6, soluble interleukin-6; MM, multiple myeloma; scFv, singlechain variable fragment; IL6(T23), N-terminal 23 amino acids deleted form of human interleukin-6; E. coli, Escherichia coli; IPTG, isopropyl- $\beta$-D-1-thiogalactopyranoside; PBS, phosphatebuffered saline; WBC, white blood cells; RBC, red blood cells; AST, aspartate aminotransferase; ALT, alanine aminotransferase; MTS, 3-(4,5-dimethylthiazol-2-yl)-5-(3-carboxymethoxyphenyl)-2(4-sulfophenyl)-2H-tetrazolium

Key words: recombinant immunotoxin, multiple myeloma, IL6(T23)-PE38KDEL, antitumor observed in 30 and $80 \%$ of mice treated intravenously and intraperitoneally, respectively, with $0.4 \mathrm{mg} / \mathrm{kg} / \mathrm{day}$ for 10 days. These results demonstrated that the recombinant immunotoxin IL6(T23)-PE38KDEL kills IL6R-overexpressing cancer cells, and causes significant tumor regression.

\section{Introduction}

Multiple myeloma (MM) is a type of malignancy characterized by clonal plasmocyte hyperplasia that exhibits multi-drug resistance, tolerance to chemotherapy and a poor therapeutic outcome (1-3). Recombinant immunotoxins (RITs) with increased antitumor specificity and reduced cellular resistance have been designed to target several groups of tumor cell surface molecules. These surface molecules include cell surface receptors, cluster designation molecules, oncofetal proteins, angiogenesis pathway proteins and tumor stromal cells (4). Attempts have been made to kill tumor cells through specific surface molecules (5). It has been found that a large variety of monoclonal antibodies (mAb) bind to antigens on cancer cells and kill cancer cells through apoptosis. However, very few antibodies are able to kill sufficient numbers of cells to cause tumor regression in experimental animals and even fewer are useful in the treatment of malignancy in humans. Therefore it is often necessary to arm the mAb with a cytotoxic ligand (6). Certain single-chain variable fragments ( $\mathrm{scFvs}$ ) have produced RITs, but the low affinity of scFvs often limits the cytotoxicity of RITs and prevents the killing of cancer cells. DAB389-IL2 and LHRH-PE40 cause complete tumor regression in some patients (7-10), and the mild side effects of these anticancer drugs are acceptable. However, a high expression of specific antigens on the tumor cell surface is extremely rare. For these reasons we re-evaluate cytokine-mediated RITs in this study.

MM cells often express over 10,000 interleukin-6 receptor (IL6R) sites/cell $(11,12)$. Overexpression of IL6R has been found not only in myeloma cells, but also on the surface of many human tumor cell lines, including hepatomas (PLC/ PRF, Hep3B and HEPG2), leukemias (HL60 and U937), prostatic carcinomas (LNCaP, DU145 and PC3), gastrointestinal cancers (BGC-823 and Caco2), and virus-transformed CESS cells $(13,14)$. However, IL6R is either absent or expressed at very low levels in normal cells $(15,16)$. IL6-PE ${ }^{4 \mathrm{E}}$ and IL6-PE40 
have been constructed to evaluate their therapeutic efficacy against a variety of IL6R-overexpressing malignancies. The two forms of RIT demonstrated significant antitumoral effects with mild hepatotoxicity for human hepatoma and acute myelocytic leukemia in mice $(17,18)$. These results indicated that, despite IL6R being expressed in several normal cell types and sIL6R being expressed in the serum, both forms of RIT have significant antitumor effects to target the IL6Roverexpressing tumor cells.

Mature human IL6 has a stable central structure that binds to IL6R, and the deletion of the N-terminal 28 amino acids in IL6 does not affect its receptor-binding activity (19-21). The most common mutant of Pseudomonas exotoxin (PE) is PE38KDEL, in which the domain Ia (amino acids 1-252) and a portion of the domain Ib (amino acids 365-380) have been deleted and in which the C-terminal amino acids 609-613 (REDLK) of PE were replaced to improve cytotoxicity by the KDEL sequence. The KDEL sequence improves the cytotoxicity of PE38 by increasing the affinity of the receptor which transports the toxin from the transreticular Golgi apparatus to the endoplasmic reticulum (ER), where it is translocated to the cytosol and kills cells by inactivating elongation factor 2 (EF2) $(22,23)$.

Based on the above theories and facts, we generated a new structure of IL6-mediated immunotoxin to kill IL6Roverexpressing tumors. This new immunotoxin was evaluated in three aspects: the specific cytotoxicity to IL6R-overexpressing $\mathrm{MM}$ cells in vitro, antitumor effects and side effects in vivo using immunocompetent mice and murine MM tumor models. The recombinant immunotoxin IL6(T23)-PE38KDEL was found to be capable of killing IL6R-overexpressing cancer cells and causing significant tumor regression.

\section{Materials and methods}

Animals and cell lines. For the cytotoxicity and antitumor assays, 6- to 8-week-old female BALB/c mice weighing 16-18 g were purchased and bred under pathogen-free conditions in the animal center of Jilin University, Changchun, China. Animal care and use were in compliance with institutional guidelines. U266 (human myeloma), SP2/0 (mouse myeloma), and CEM (T lymphoid leukemia) cells were purchased from the Tumor Cell Bank at the Chinese Academy of Medical Sciences.

Design and synthesis of a recombinant toxin gene. The target toxin IL6(T23)-PE38KDEL is a fusion protein that was constructed by connecting human IL6 missing N-terminal 23 amino acids to PE38KDEL. The IL6(T23)-PE38KDEL gene sequence was encoded using $E$. coli preferred codons by Jcat software (24). The complete gene sequence with restriction enzyme sites $N c o$ I and XhoI was synthesized, cloned into the pUC57 vector and then transformed into the E. coli DH5 $\alpha$ strains by the Shanghai Sangon Biotechnology Company.

Expression and purification of recombinant protein. The gene fragment encoding IL6(T23)-PE38KDEL was inserted between the $N c o I$ and $X h o I$ sites of the expression vector pET28a(+) plasmid (Novagen). The recombinant plasmid carrying the fusion gene was expressed via isopropyl- $\beta$-D-1thiogalacto-pyranoside (IPTG) induction in the E. coli BL21
( $\lambda$ DE3) cells. The expressed product was purified from the inclusion bodies of the bacterial cells as previously described $(25,26)$, with slight modifications. In brief, following sonication and centrifugation of the bacterial cells, the inclusion bodies were washed extensively with $2.5 \%$ Triton X-100 and TE buffer and then dissolved, denatured and reduced in guanidinedithioerythritol solution. Following denaturation, the inclusion body protein was refolded by dilution in a renaturation buffer containing arginine and reduced glutathione to facilitate redox shuffling and then concentrated through a Millipore Amicon concentrator $(30 \mathrm{kDa})$. The solution containing the refolded protein was centrifuged, filtered, and applied onto a 20-ml Q HP column attached to a fast protein liquid chromatography system ÄKTA Explorer 100, then washed with $20 \mathrm{mM}$ Tris-Cl, $\mathrm{pH} 7.4$, and eluted with a stepwise gradient of $20 \mathrm{mM}$ Tris- $\mathrm{Cl}$, $\mathrm{pH} 7.4$, containing $1.0 \mathrm{M} \mathrm{NaCl}$. The fraction containing the peak cytotoxic activity was then diluted 3-fold with $20 \mathrm{mM}$ Tris-Cl, pH 7.4, and loaded onto an 8-ml monoQ column, which was then eluted with a linear gradient to obtain the cytotoxicity protein. After removing endotoxin through a polymyxin B column, the concentrated monoQ-purified protein was loaded on a Superdex 200 column and eluted with phosphate-buffered saline (PBS). The single elution peak was collected and saved. For the in vitro and in vivo studies, a batch of active IL6(T23)PE38KDEL was produced with a low endotoxin content. The recombinant immunotoxin IL6-PE40 was prepared and identified as described (11). The protein concentration of the purified chimeric toxins was determined by the Bradford assay (27). The endotoxin concentrations of RIT were determined by a colorimetric Limulus test (28).

In vitro cytotoxicity assay. The number of IL6Rs in cells was measured by IL6 receptor-binding assays (13). The specific cytotoxicity of IL6(T23)-PE38KDEL and IL6-PE40 was assessed in triplicate using two IL6R-positive cell lines, U266 and SP2/0, and one IL6R-negative cell line, CEM, by MTS colorimetric assay (29). Briefly, the cells were washed three times with RPMI-1640 medium to remove autocrine IL6 and seeded in a 96-well cell culture plate at $1 \times 10^{4}$ cells/well (200 $\mu \mathrm{l}$ ). Following $0.22 \mu \mathrm{m}$ membrane filtration, sterilization and dilution in PBS containing $0.2 \%$ serum albumin, various concentrations of IL6(T23)-PE38KDEL (20 $\mu \mathrm{l})$ were added to the cell suspension. After adding RIT, the cells were incubated at $37^{\circ} \mathrm{C}$ for $30 \mathrm{~h}$ and $20 \mu \mathrm{l} /$ well MTS/PMS was added. After $3 \mathrm{~h}$ the plates were read at $490 \mathrm{~nm}$ using a microreader.

For competition experiments, various amounts of recombinant IL6 were added $20 \mathrm{~min}$ before IL6(T23)-PE38KDEL (40 ng/ml) was added to U266 and SP2/0 cells. The growth inhibition ratios of tumor cells were measured by MTS assay. For the morphological observation of IL6(T23)-PE38KDEL cytotoxicity, U266, SP2/0 and CEM cells were plated in a 96-well cell culture plate. After $4 \mathrm{~h}$ incubation, $50 \mathrm{ng} / \mathrm{ml}$ IL6(T23)-PE38KDEL was added to the cells for $30 \mathrm{~h}$. The control groups were seeded and cultured in the same conditions but without IL6(T23)-PE38KDEL.

Toxicity and maximum tolerated dose in mice. Groups of 10 female BALB/c mice were given single or multiple (QD for 10 days) injections of increasing doses of IL6(T23)PE38KDEL intravenously (i.v.) through the tail vein, and the 
animals were observed over 2 weeks. The $\mathrm{LD}_{50}$ was calculated using the Trimmed Spearman-Karber program. The maximum cumulative tolerated dose of IL6(T23)-PE38KDEL in mice was determined. Side effects were determined by autopsy and histopathology in the animals administered with lethal levels of RIT.

To determine the side effects in liver and renal cells, and the effect on the number of peripheral blood cells following treatment, a group of mice receiving the high dose of IL6(T23)PE38KDEL (0.4 mg/kg/day for 10 days) was sacrificed 10 days after the treatment. Whole blood was collected in heparinized tubes and the differential blood cells were counted from each mouse in quadruplicate using a hemocytometer. Plasma was collected following centrifugation and kept frozen at $-20^{\circ} \mathrm{C}$. Biochemical parameters were analyzed and measured with a multi-test analyzer.

In vivo antitumor assay. The antitumor activity of IL6(T23)PE38KDEL was evaluated in female BALB/c mice previously injected with MM cells. SP2/0 cells $\left(1 \times 10^{7}\right)$ were injected intraperitoneally (i.p.) into the mice and the development of MM was monitored by body weight and the serum paraprotein level (30). Five days after the injection (DPI) of the MM cells, the mice were randomly divided into 4 groups of 10 mice each. Three groups received a daily injection of IL6(T23)PE38KDEL in $0.1 \mathrm{ml}$ PBS at doses of $0.1,0.2$ and $0.4 \mathrm{mg} / \mathrm{kg} /$ day for 10 days by i.v. through the tail vein. The control group received the corresponding volume of PBS in the same manner. Mice that died during the treatment with IL6(T23)-PE38KDEL were subjected to histopathological examination. Differences in survival time of the experimental animals were evaluated with a log-rank test and Kaplan-Meier survival curves. $\mathrm{P}<0.05$ was considered to indicate a statistically significant difference. To study the effect of the method of IL6(T23)-PE38KDEL administration, one group of MM-treated mice (10 mice) received a daily i.p. injection of IL6(T23)-PE38KDEL at a dose of $0.4 \mathrm{mg} / \mathrm{kg} /$ day for 10 days. The control group received the same volume of PBS in the same manner. The mice were sacrificed on day 15 and dissected to determine the effect of interventional therapy. Whole blood was collected, and the main physiological and biochemical parameters of the blood were measured.

\section{Results}

Expression and purification of IL6(T23)-PE38KDEL. For the preparation of IL6(T23)-PE38KDEL, the transformed E. coli cells were induced by IPTG at $37^{\circ} \mathrm{C}$ for $4 \mathrm{~h}$, then collected and processed for the extraction of RIT from inclusion bodies. Following denaturation and reduction, the active RIT was purified from the refolding solution by ion exchange, polymyxin B endotoxin removal and gel filtration chromatography. The RIT accumulated in E. coli cells at approximately $20 \%$ of total protein as estimated from the densitometric scanning of Coomassie blue-stained SDS-PAGE gels. Active IL6(T23)PE38KDEL was eluted from a Q column with a Tris-HCl buffer containing $0.20 \mathrm{M} \mathrm{NaCl}$. The final purified protein showed a single band of $56 \mathrm{kDa}$ that reacted specifically with rabbit antiPE38 antibodies as indicated by western blot analysis (Fig. 1). The endotoxin content of RIT was $2.3 \mathrm{EU} / \mathrm{mg}$.

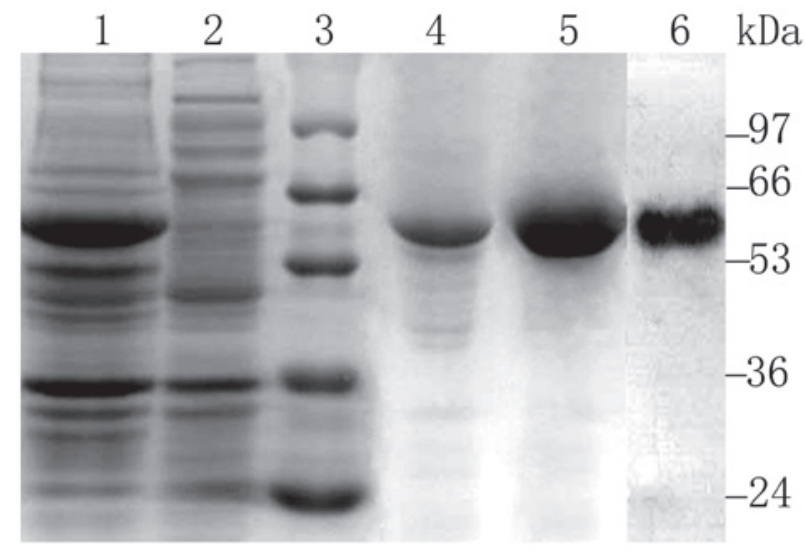

Figure 1. SDS-PAGE analysis of IL6(T23)-PE38KDEL. IL6(T23)PE38KDEL was expressed and purified. SDS-PAGE (10\%) showed that the target protein at approximately $56 \mathrm{kDa}$ could be detected using Coomassie blue staining and immunoblotting. Lane 1, pellet from cell lysate induced with IPTG; lane 2, supernatant from cell lysate induced with IPTG; lane 3 , middle molecular weight standard marker; lane 4, inclusion body protein; lane 5, purified IL6(T23)-PE38KDEL (5 $\mu \mathrm{g}$ ); lane 6, immunoblot of $0.5 \mu \mathrm{g}$ pure IL6(T23)-PE38KDEL using anti-PE monoclonal antibody.

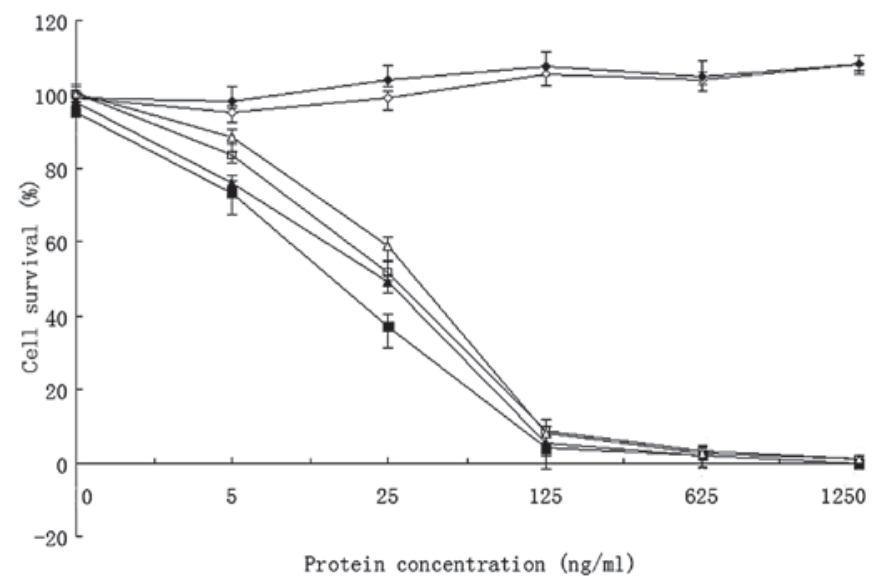

Figure 2. Cytotoxic activity of IL6(T23)-PE38KDEL and IL6-PE40 in U266, SP2/0 and CEM cells. Recombinant IL6(T23)-PE38KDEL and IL6-PE40 were added at various concentrations to the cells $\left(1.0 \times 10^{4}\right.$ cells/well), and cell viability was evaluated after $30 \mathrm{~h}$ by MTS assay. U266 (๓) and SP2/0 (ی) cells were sensitive to IL6(T23)-PE38KDEL with IC $_{50}$ values of 20 and $25 \mathrm{ng} / \mathrm{ml}$, respectively. U266 ( $\square)$ and SP2/0 $(\triangle)$ cells were sensitive to IL6-PE40 with $\mathrm{IC}_{50}$ values of 39 and $46 \mathrm{ng} / \mathrm{ml}$, respectively. CEM $(\diamond)(\diamond)$ cells were insensitive to IL6(T23)-PE38KDEL and IL6-PE40, respectively.

Cytotoxic specificity. Several control experiments were performed to determine whether the cytotoxicity of IL6(T23)PE38KDEL was specific and required binding to IL6R by IL6(T23). In vitro cytotoxic studies revealed that U266 with 15,500 IL6R sites/cell and SP2/0 with 16,500 IL6R sites/ cell were highly sensitive to IL6(T23)-PE38KDEL and IL6-PE40 (Fig. 2). The IC $_{50}$ values of IL6(T23)-PE38KDEL were 20 and $25 \mathrm{ng} / \mathrm{ml}$, respectively. IL6(T23)-PE38KDEL was 2.0-fold more active than IL6-PE40 in U266 cells, and IL6(T23)-PE38KDEL was 1.8-fold more toxic than IL6-PE40 in SP2/0 cells. Conversely, the IL6R-negative CEM cells were unresponsive to IL6(T23)-PE38KDEL up to $1,000 \mathrm{ng} / \mathrm{ml}$. The cytotoxicity of IL6(T23)-PE38KDEL was effectively blocked 
Table I. Toxicity of IL6(T23)-PE38KDEL administered to mice by intravenous injection.

\begin{tabular}{llc}
\hline Dose $(\mathrm{mg} / \mathrm{kg})$ & Treatment schedule & Mortality \\
\hline 0.25 & Single dose & $0 / 10$ \\
0.50 & Single dose & $0 / 10$ \\
0.75 & Single dose & $3 / 10$ \\
1.00 & Single dose & $4 / 10$ \\
1.25 & Single dose & $7 / 10$ \\
1.50 & Single dose & $10 / 10$ \\
0.50 & Daily for 10 doses & $2 / 10$ \\
0.40 & Daily for 10 doses & $0 / 10$ \\
\hline
\end{tabular}

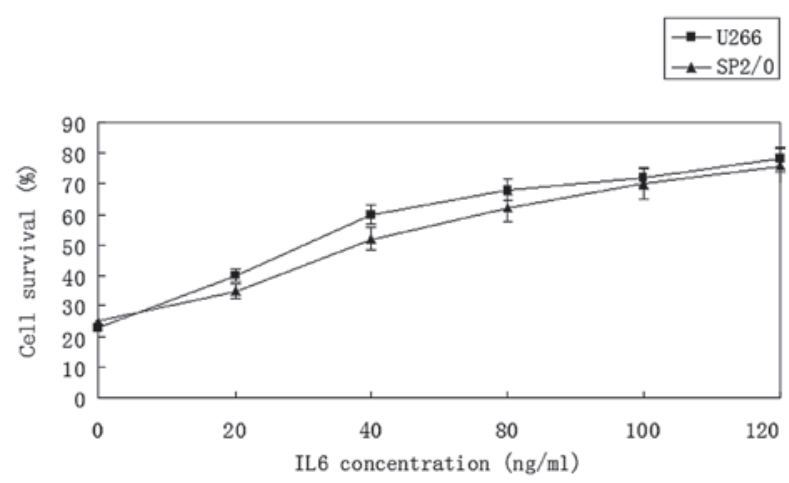

Figure 3. Inhibition of cytotoxic activity of IL6(T23)-PE38KDEL. IL6(T23)PE38KDEL competed with various amounts of recombinant IL6 in U266 (or SP2/0 (₫) cells. Cytotoxicity of IL6(T23)-PE38KDEL could be blocked by excess IL6. The results showed that the cytotoxic activity of IL6(T23)PE38KDEL in U266 and SP2/0 cells was specifically mediated by the IL6 receptor.

by IL6 (Fig. 3). Compared with the control groups, U266 and SP2/0 cells treated with IL6(T23)-PE38KDEL demonstrated shrinkage, cytoplasmic dark granularity and death. As the negative control cells, CEM cells were not affected after $30 \mathrm{~h}$ treatment with IL6(T23)-PE38KDEL (Fig. 4). The results indicated that IL6(T23)-PE38KDEL had better activity and was able to specifically bind to the human and mouse IL6R, and be targeted to kill IL6R-positive cells.

Non-specific toxicity and treatment doses of IL6(T23)PE38KDEL in mice. The non-specific toxicity and treatment doses of IL6(T23)-PE38KDEL were evaluated in normal mice. Groups of 10 mice received a one-time i.v. injection with varying doses of RIT and were observed for 2 weeks. The mortalities occurred within $72 \mathrm{~h}$ of the injection (Table I). The $\mathrm{LD}_{50}$ of IL6(T23)-PE38KDEL was $0.985 \mathrm{mg} / \mathrm{kg}(95 \%$ confidence range, $0.839-1.138 \mathrm{mg} / \mathrm{kg}$ ), and was calculated with SPSS Statistics 17.0. A single dose of $1 \mathrm{mg} / \mathrm{kg}$ IL6(T23)PE38KDEL by i.v. injection (100 $\mu \mathrm{l})$ was lethal to $40 \%$ of the mice. A single dose of $0.5 \mathrm{mg} / \mathrm{kg}$ IL6(T23)-PE38KDEL was not lethal, but $0.5 \mathrm{mg} / \mathrm{kg} / \mathrm{day}$ for 10 days was lethal to $20 \%$ of the mice. However, treatment with a total IL6(T23)PE38KDEL dose up to $4 \mathrm{mg} / \mathrm{kg}(0.4 \mathrm{mg} / \mathrm{kg} /$ day for 10 days $)$ was not lethal. Higher doses of IL6(T23)-PE38KDEL caused acute death of experimental mice, and the autopsy results showed

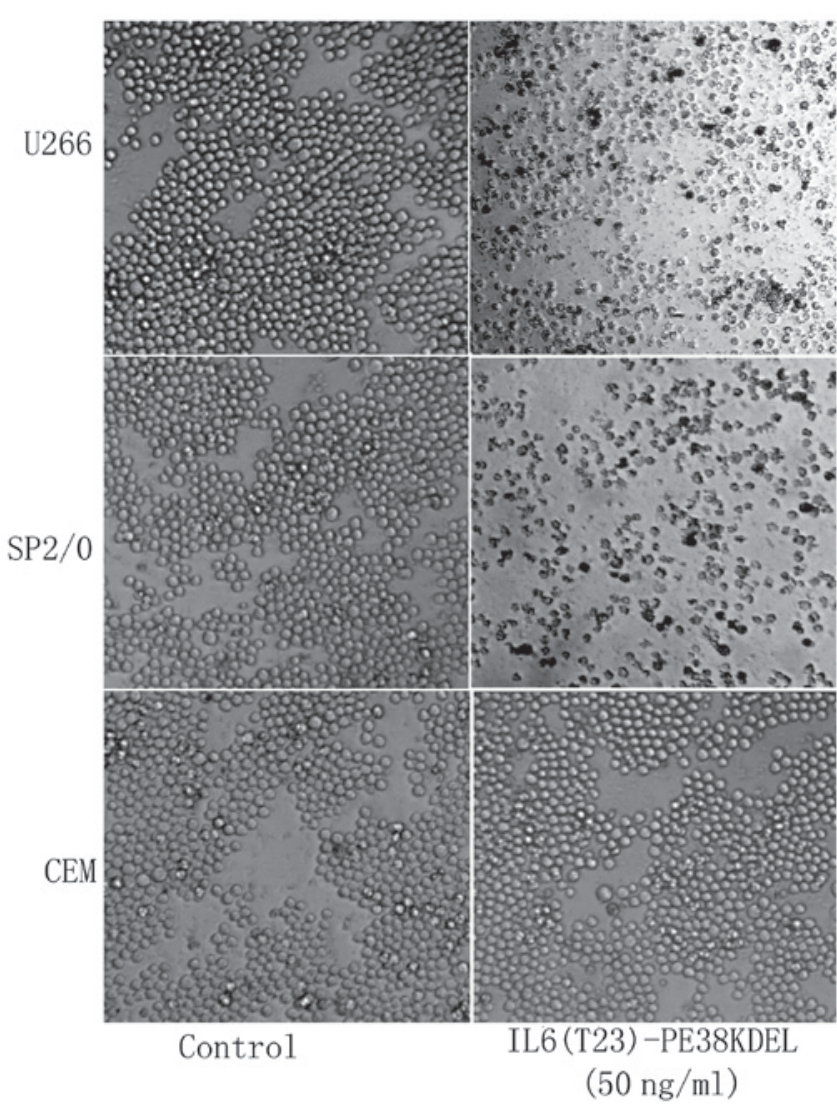

Figure 4. Light micrographs of U266 and SP2/0 controls and cells treated with $50 \mathrm{ng} / \mathrm{ml}$ IL6(T23)-PE38KDEL for $30 \mathrm{~h}$. U266 and SP2/0 cells were killed by IL6(T23)-PE38KDEL, but CEM cells were not damaged.

that the dose-limiting toxicity of IL6(T23)-PE38KDEL was hepatotoxicity as the major cause of death.

To determine the toxic effects of IL6(T23)-PE38KDEL in normal tissues, normal mice were treated for 10 days continuously by i.v. injection $(0.4 \mathrm{mg} / \mathrm{kg} / \mathrm{day})$. Treatment with IL6(T23)-PE38KDEL had no effect on the absolute number of white blood cells (WBC), but led to increased platelet numbers and a slight increase in granulocyte levels, indicative of inflammatory reactions in the body (Table II). The red blood cell (RBC) and hematocrit levels decreased during the treatment. The levels of hepatic enzymes aspartate aminotransferase (AST), alanine aminotransferase (ALT) and alkaline phosphatase (ALP) were elevated to approximately twice those of the untreated mice, while the renal function was unchanged by the BUN and CK assay (Table II). These data indicated that a RIT dose of $0.4 \mathrm{mg} / \mathrm{kg} /$ days for 10 days was well-tolerated with mild hepatotoxicity.

Antitumor activity in mice bearing multiple myeloma. Following injection of SP2/0 cells into BALB/c mice, all animals developed MM, characterized by an increase in serum paraprotein and mild abdominal swelling. The survival time assay was used as a parameter to evaluate the therapeutic efficacy of IL6(T23)-PE38KDEL. Following inoculation of $1 \times 10^{7}$ cells, untreated MM lead to the gradual death of the negative controls from day 16 to 19 . The gross pathological findings were large abdominal tumor masses causing ascites and hepatosplenomegaly, and large numbers of tumor metastases were 
Table II. Effect of IL6(T23)-PE38KDEL on physiological and biochemical parameters of blood after 10 days of treatment.

\begin{tabular}{lccc}
\hline Items & Control group & Intravenous group & Intervention group \\
\hline WBC $\left(10^{6} / \mathrm{ml}\right)$ & $8.61 \pm 2.06$ & $8.99 \pm 2.20$ & $8.94 \pm 2.14$ \\
RBC $\left(10^{9} / \mathrm{ml}\right)$ & $8.90 \pm 1.05$ & $7.2 \pm 1.37$ & $7.99 \pm 0.85$ \\
Platelets $\left(10^{9} / \mathrm{l}\right)$ & $1246 \pm 175$ & $1765 \pm 189$ & $1365 \pm 206$ \\
Hematocrit $(\%)$ & $48.0 \pm 2.0$ & $36.0 \pm 3.0$ & $45 \pm 5.0$ \\
Neutrophil $(\%)$ & $40.0 \pm 9$ & $73.4 \pm 11$ & $63 \pm 10$ \\
AST $(\mathrm{U} / \mathrm{l})$ & $112.70 \pm 19.45$ & $191.53 \pm 18.38$ & $139.83 \pm 16.84$ \\
ALT $(\mathrm{U} / \mathrm{l})$ & $40.08 \pm 14.63$ & $96.72 \pm 16.80$ & $55.80 \pm 14.66$ \\
ALP $(\mathrm{U} / \mathrm{l})$ & $88.80 \pm 6.05$ & $173.45 \pm 14.51$ & $120.90 \pm 11.83$ \\
BUN $(\mathrm{mM})$ & $8.42 \pm 1.30$ & $8.50 \pm 0.97$ & $8.31 \pm 1.53$ \\
Creatine kinase $(\mathrm{mM})$ & $84.70 \pm 18.40$ & $81.62 \pm 17.35$ & $83.15 \pm 19.86$ \\
\hline
\end{tabular}

WBC, white blood cells; RBC, red blood cells; AST, aspartate aminotransferase; ALT, alanine aminotransferase; ALP alkaline phosphatase; BUN, blood urea nitrogen.

A

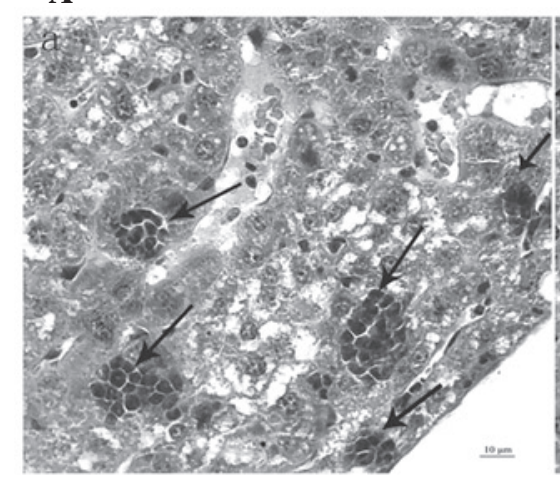

B

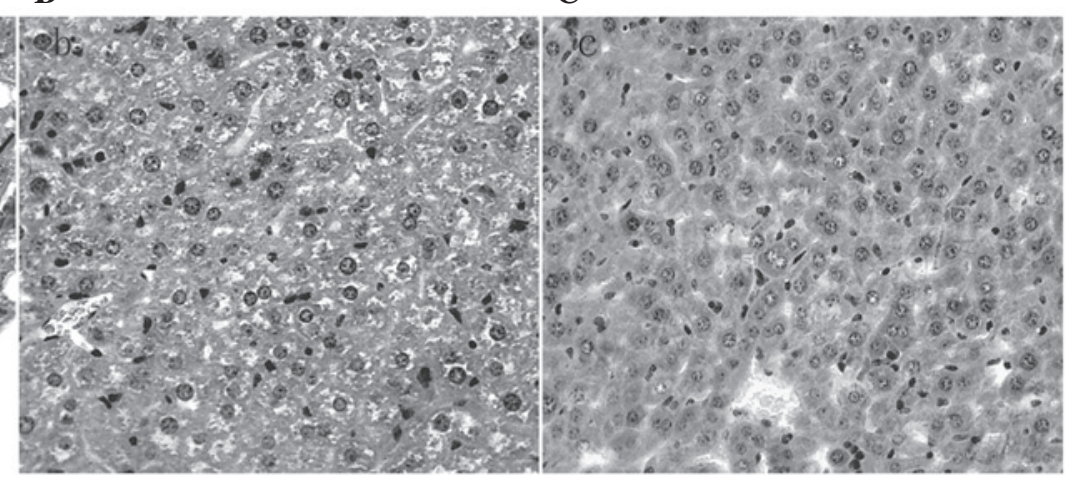

Figure 5. Light micrograph of liver tissue from a treated mouse, control and normal mouse. (A) Light micrograph of liver tissue from an untreated control. A large number of MM metastases appeared in the liver tissue of the control (10x40). The typical tumor metastases (thin arrows) grew in the vicinity of hepatic sinusoid. (B) Light micrograph of liver biopsy of a mouse treated with interventional procedures demonstrated an essentially normal appearance of hepatocytes, with no evident tumor metastases (10x40). (C) Light micrograph of a normal mouse liver tissue.

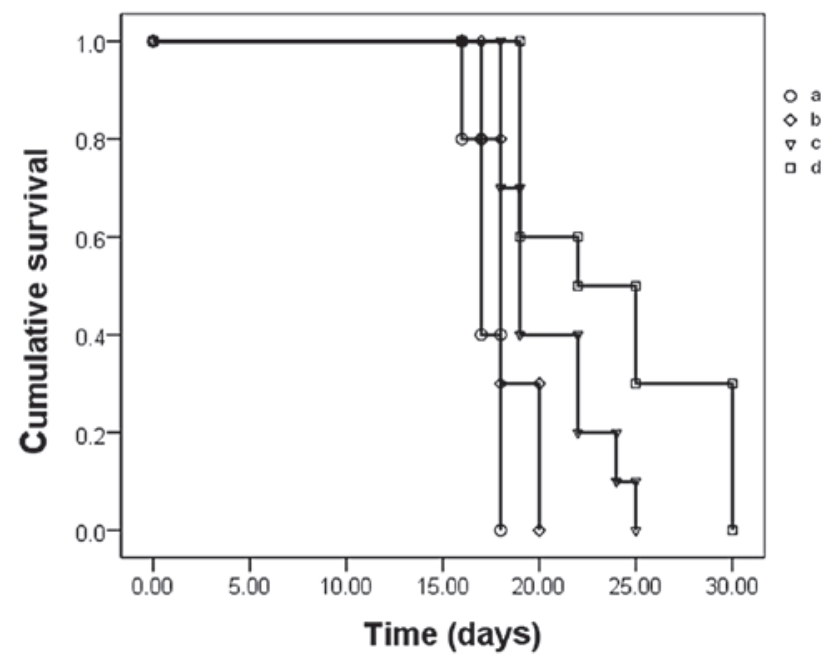

Figure 6. Survival curves of MM mice receiving IL6(T23)-PE38KDEL at various dose levels. On day 0 mice were injected with $1.0 \times 10^{7}$ multiple myeloma (MM) cells. A 10-day treatment with IL6(T23)-PE38KDEL was started on day 5 at dose levels of $0.4 \mathrm{mg} / \mathrm{kg} / \mathrm{day}$ (line d), $0.2 \mathrm{mg} / \mathrm{kg} / \mathrm{day}$ (line c) and $0.1 \mathrm{mg} / \mathrm{kg} / \mathrm{day}$ (line b). The control (line a) received PBS only. The survival time of the experimental animals with the three different doses increased by $6.8,3.2$ and 1.2 days, respectively, compared with the control group. found in the liver tissue (Fig. 5A). Five days after inoculation of the SP2/0 cells, tumor-bearing mice were treated with three doses of IL6(T23)-PE38KDEL (0.4, 0.2 and $0.1 \mathrm{mg} / \mathrm{kg} / \mathrm{day})$ to determine the anti-myeloma effects. The survival time of animals treated with the three different doses increased by 1.2, 3.2 and 6.8 days, respectively, when compared to PBS-treated MM controls $(\mathrm{p}=0.002$, Kaplan-Meier survival analysis; Fig. 6). In the $0.4 \mathrm{mg} / \mathrm{kg} / \mathrm{day}$ group, 3 of the 10 mice were alive and in good condition on day 30 . When the animals were sacrificed and autopsied, we found that the three mice had no visible intra-abdominal tumors. These results demonstrated that IL6(T23)-PE38KDEL significantly increased the survival times of treated mice and exhibited a significant dose-dependent antitumor effect against MM mice.

In a separate experiment, the treatment of MM mice with $0.4 \mathrm{mg} / \mathrm{kg} /$ day of IL6(T23)-PE38KDEL for 10 days (from day 5 to 15) by i.p injection led to a notable effect of tumor regression. When the mice were sacrificed and dissected, we observed that 8 of the 10 mice had no visible tumors in the abdominal cavity, and two mice had significantly smaller tumors than the control group. Compared to the control group, the liver histopathology from mice treated with interven- 
tional injections revealed an essentially normal appearance of hepatocytes, without evident tumor metastases (Fig. 5B). The control group had large tumors that filled the abdominal cavity, accompanied with large amounts of ascites. The complete regression rate of IL6(T23)-PE38KDEL in the mice was significantly higher by interventional injection compared to i.v. administration.

The blood assay (Table II) revealed that IL6(T23)PE38KDEL did not markedly affect the main physiological parameters of blood, but caused a slight increase in liver enzyme activity. Compared with the normal mice treated, the liver enzyme levels of MM mice treated were significantly lower.

\section{Discussion}

The present study aimed to evaluate the antitumor activity of the recombinant IL6(T23)-PE38KDEL in vitro and in vivo. The IL6(T23)-PE38KDEL gene using E . coli preferred codons was designed to overcome the bottleneck caused by the low expression level of the natural gene in the pET expression system. Codon optimization has become a very effective means to improve protein expression $(24,31,32)$. In a previous study, we constructed the IL6(T23)-PE38KDEL gene by overlap PCR using the natural IL6 and PE genes. We attempted to use the pET28a, pET22b, pQE30 and pKK322 vectors expressing the toxin in E. coli, but the expression level was only $2-6 \%$ of the total protein concentration (unpublished data). The expression level of the codon-optimized IL6(T23)-PE38KDEL gene was 5- to 6-fold that of the natural gene. The natural IL6(T23)PE38KDEL gene contains a large number of rare codons, thus we reasoned that it was unsuitable for expressing in E. coli. As an outcome, the recombinant IL6(T23)-PE38KDEL was expressed and accumulated at approximately 20-24\% of total protein by codon optimization.

The cytotoxic mechanism of cytokine-PE toxins has previously been elaborated (7,18,33-35). The cytokine-PE chimeric toxins and derivatives bind to cytokine receptors on the cell surface and then enter the cell through receptormediated endocytosis. In the endocytic compartment, it is cleaved into two fragments by a furin enzyme. The C-terminal fragment, which is composed of PE domain III and a portion of domain II, is transported to the ER through the KDEL sequence (KDEL functions as a REDL ER-targeting motif sequence for ER retrieval). The C-terminal fragment then translocates to the cytosol and enzymatically inactivates EF2, causing the inhibition of protein synthesis and leading to cell death. Therefore, the cytotoxicity of cytokine-PE chimeric proteins depends not only on the cell receptor number, but also on binding affinity, rate of internalization, rate of processing into an active form and rate of translocation into the cytosol (6). The hepatoma cell line SK-HEP with approximately 100 IL6R sites/cell and the prostatic carcinoma cell line PC3 with 400 IL6R sites/cell were both insensitive to IL6-PE ${ }^{4 E}$ and IL6-PE40 (13). The cleavage of PE-derived immunotoxins by furin is a rate-limiting step for cytotoxic activity (36). IL6R is either absent or present at very low levels in normal cells $(11,12)$, and furin is widely expressed at very low levels in normal tissues (37). Conversely, IL6, IL6R and furin are overexpressed in certain malignancies (16,37-39). The key factors of competitive inhibition of IL6 and very low levels of IL6R and furin limit the efficacy of IL6(T23)-PE38KDEL to bind to the normal cell surface and be internalized and processed; this results in insufficient catalytic fragments to kill normal cells. According to the functional characteristics of tIL6 (40) and IL6-PE40 ${ }^{\mathrm{Asp553}}$ (11), and the structural features of IL6 $(15,41)$, we reasoned that, as with the IL6 ligand, IL6(T23) only has a receptor-binding function, and loses most of its IL6 transsignaling functionality due to truncation and PE conjugation.

The cytotoxicity of IL6(T23)-PE38KDEL was greater than that of IL6-PE40 in vitro. The main reason for the increased activity may be that PE40 was transformed into PE38KDEL. In addition, the recombinant immunotoxin exhibited specific antitumor activity against both human IL6R and murine IL6R. The ID50 value showed that the sensitivity of U266 cells to IL6(T23)-PE38KDEL was slightly higher than that of SP2/0, therefore we adapted the mouse SP2/0 tumor model to evaluate the antitumor effects and side effects of IL6 (T23)-PE38KDEL. The repeat administration of IL6(T23)-PE38KDEL by i.v. injection, at a dose of $0.4 \mathrm{mg} / \mathrm{kg} /$ day for 10 days, resulted in a $30 \%$ tumor regression and a significant increase in the survival time. IL6(T23)-PE38KDEL (0.4 mg/kg/day for 10 days) by i.p. injection resulted in a notable response of $80 \%$ tumor regression. The complete regression rate using i.v. administration was significantly lower than that using interventional injection; this result indicated that certain factors in the blood acted as a counteractant which cleared IL6(T23)-PE38KDEL before binding to the tumor cells. Our data indicated that IL6(T23)PE38KDEL may be suitable to target tumors by interventional therapy. MM liver metastases may be associated with IL6. In the MM mouse model, a large amount of myeloma cell growth was bound to produce a large amount of IL6 in an autocrine manner. The pleiotropic cytokine IL6, a major mediator of inflammation (42) and an activator of STAT3 (43), may serve to promote the dissemination of myeloma cells into the blood circulation, and planting and developing in the liver. Even if there is competitive inhibition between IL6 and IL6(T23)PE38KDEL, sufficient IL6(T23)-PE38KDEL binding to IL6R kills IL6R-bearing cancer cells and inhibits tumor metastasis. The liver pathology revealed that MM liver metastasis in the high dose group was significantly lower than that in the control group. Therefore, we suggest that IL6(T23)-PE38KDEL may be suitable for use after surgery to inhibit IL6R-bearing tumor cell metastasis.

Since human IL6 crossreacts with murine IL6R, we used normal mice to evaluate the side effects of IL6(T23)PE38KDEL. The results indicated that IL6(T23)-PE38KDEL did not markedly affect the absolute WBC and RBC numbers, which indicates that IL6(T23)-PE38KDEL does not kill the myeloid progenitor cells. In normal mice, the high dose of IL6(T23)-PE38KDEL caused mild liver cell damage. Such hepatotoxicity may be attributed to non-specific internalization of the drug or lower IL6R levels in liver cells $(44,45)$. However, since the nonspecific toxic effects were significantly reduced in the tumor-bearing mice treated, it may be the large amount of IL6(T23)-PE38KDEL binding to IL6-overexpressing on tumor that causes the reduction of IL6(T23)-PE38KDEL concentration in liver, and results in lower liver toxicity.

Hepatotoxicity of IL6(T23)-PE38KDEL was the major cause of death in the treated mice. Similar results have also 
been observed in mice treated with IL6-PE ${ }^{4 \mathrm{E}}$ and IL2-PE ${ }^{4 \mathrm{E}}$ $(26,46)$. However, hepatotoxicity of PE-derived immunotoxins is commonly dose-limiting in mice, but only rarely in patients (7). By comparing the ID50 value in mice, the side effects of IL6(T23)-PE38KDEL were lower than those of IL6-PE ${ }^{4 \mathrm{E}}$, which may be attributed to the conversion of $\mathrm{PE}^{4 \mathrm{E}}$ to PE38KDEL. Certain studies have shown that high-dose injection of IL6 induces anemia and leads to a reduced RBC in mice (47-49).

The antitumor activity of IL6(T23)-PE38KDEL depends not only on the IL6R number, but also on the levels of IL6 and sIL6R. High levels of IL6 and sIL6R in the blood have been found in malignant conditions $(12,50,51)$. The antitumor activity of IL6(T23)-PE38KDEL can be blocked by an excess of IL6 and sIL6R. Therefore, we cannot rule out the possibility that IL6 and sIL6R act as competitors for IL6(T23)-PE38KDEL, which leads to the poor effects observed in the low-dose group. Specifically, sIL6R binds to the toxin forming complexes that may lead to non-specific effects. IL6(T23)-PE38KDEL is a protein antigen in mice, and long-term injection of the toxin is likely to produce neutralizing antibodies to decrease the antitumor activity. However, more studeis are required to identify whether polyethylene glycol modification could decrease the side effects and immunogenicity of IL6(T23)-PE38KDEL(52).

In conclusion, evaluation of IL6(T23)-PE38KDEL indicated that the recombinant toxin has selective cytotoxicity against IL6R-overexpressing cancer cells in vitro and in vivo. At a dose of $0.4 \mathrm{mg} / \mathrm{kg} /$ day for 10 days, IL6(T23)-PE38KDEL caused significant tumor regression in mice. These results make IL6(T23)-PE38KDEL a potential candidate for further development as an anticancer drug for IL6R-overexpressing tumors.

\section{Acknowledgements}

We would like to thank Mrs. Wang Xin Rui and Miss Peng Chao for their technical assistance, and to Li Le and Yan Dong Ming for their assistance with animal care and management. We also thank Dr Yu Lu for his revision of this manuscript.

\section{References}

1. Durie BG: Role of new treatment approaches in defining treatment goals in multiple myeloma - the ultimate goal is extended survival. Cancer Treat Rev 36: S18-S23, 2010.

2. Shain KH and Dalton WS: Environmental-mediated drug resistance: a target for multiple myeloma therapy. Expert Rev Hematol 2: 649-662, 2009.

3. Rajkumar SV: Treatment of multiple myeloma. Nat Rev Clin Oncol 8: 479-491, 2011.

4. Mohindru M and Verma A: Engineered antibodies act as targeted therapies in cancer treatment. Indian J Pediatr 72 943-947, 2005.

5. Binyamin L, Borghaei $\mathrm{H}$ and Weiner LM: Cancer therapy with engineered monoclonal antibodies. Update on cancer therapeutics 1: 147-157, 2006.

6. Pastan I: Immunotoxins containing Pseudomonas exotoxin A: a short history. Cancer Immunol Immunother 52: 338-341, 2003.

7. Kreitman RJ: Recombinant immunotoxins containing truncated bacterial toxins for the treatment of hematologic malignancies. BioDrugs 23: 1-13, 2009.

8. Li J and Zhang JK: LHRH-PE40-induced vascular leak syndrome. Toxicol Mech Methods 16: 473-476, 2006.

9. Li J, Sun Y and Zhang J: A recombinant protein LHRH-PE40 for tumour therapy: preclinical safety studies. Basic Clin Pharmacol Toxicol 99: 398-404, 2006.
10. Kreitman RJ: Recombinant immunotoxins for the treatment of chemoresistant hematologic malignancies. Curr Pharm Des 15: 2652-2664, 2009.

11. Siegall CB, Chaudhary VK, FitzGerald DJ and Pastan I: Cytotoxic activity of an interleukin 6-Pseudomonas exotoxin fusion protein on human myeloma cells. Proc Natl Acad Sci USA 85: 9738-9742, 1988.

12. Kreitman RJ, Siegall CB, FitzGerald DJ, Epstein J, Barlogie B and Pastan I: Interleukin-6 fused to a mutant form of Pseudomonas exotoxin kills malignant cells from patients with multiple myeloma. Blood 79: 1775-1780, 1992.

13. Siegall CB, Schwab G, Nordan RP, FitzGerald DJ and Pastan I: Expression of the interleukin 6 receptor and interleukin 6 in prostate carcinoma cells. Cancer Res 50: 7786-7788, 1990.

14. Siegall CB, FitzGerald DJ and Pastan I: Cytotoxicity of IL6-PE40 and derivatives on tumor cells expressing a range of interleukin 6 receptor levels. J Biol Chem 265: 16318-16323, 1990.

15. Simpson RJ, Hammacher A, Smith DK, Matthews JM and Ward LD: Interleukin-6: structure-function relationships. Protein Sci 6: 929-955, 1997.

16. Hong DS, Angelo LS and Kurzrock R: Interleukin-6 and its receptor in cancer: implications for translational therapeutics. Cancer 110: 1911-1928, 2007.

17. Siegall CB, Kreitman RJ, FitzGerald DJ and Pastan I: Antitumor effects of interleukin 6-Pseudomonas exotoxin chimeric molecules against the human hepatocellular carcinoma, PLC/ PRF/5 in mice. Cancer Res 51: 2831-2836, 1991.

18. Rozemuller H, Rombouts WJ, Touw IP, et al: Treatment of acute myelocytic leukemia with interleukin-6 Pseudomonas exotoxin fusion protein in a rat leukemia model. Leukemia 10: 1796-1803, 1996.

19. Proudfoot AE, Brown SC, Bernard AR, Bonnefoy JY and Kawashima EH: Recombinant human IL-6 expressed in E. coli undergoes selective $\mathrm{N}$-terminal degradation: evidence that the protein consists of a stable core and a nonessential flexible N-terminal. J Protein Chem 12: 489-497, 1993.

20. Hammacher A, Ward LD, Weinstock J, Treutlein H, Yasukawa K and Simpson RJ: Structure-function analysis of human IL-6: identification of two distinct regions that are important for receptor binding. Protein Sci 3: 2280-2293, 1994.

21. Ehlers M, Grötzinger J, deHon FD, et al: Identification of two novel regions of human IL- 6 responsible for receptor binding and signal transduction. J Immunol 153: 1744-1753, 1994.

22. Weldon JE and Pastan I: A guide to taming a toxin - recombinant immunotoxins constructed from Pseudomonas exotoxin A for the treatment of cancer. FEBS J 278: 4683-4700, 2011.

23. Kreitman RJ and Pastan I: Importance of the glutamate residue of KDEL in increasing the cytotoxicity of Pseudomonas exotoxin derivatives and for increased binding to the KDEL receptor. Biochem J 307 (Pt 1): 29-37, 1995.

24. Grote A, Hiller K, Scheer M, et al: JCat: a novel tool to adapt codon usage of a target gene to its potential expression host. Nucleic Acids Res 33: W526-531, 2005.

25. Brinkmann U, Pai LH, FitzGerald DJ, Willingham $M$ and Pastan I: B3(Fv)-PE38KDEL, a single-chain immunotoxin that causes complete regression of a human carcinoma in mice. Proc Natl Acad Sci USA 88: 8616-8620, 1991.

26. Kreitman RJ and Pastan I: Purification and characterization of IL6-PE4E, a recombinant fusion of interleukin 6 with Pseudomonas exotoxin. Bioconjug Chem 4: 581-585, 1993.

27. Olson BJ and Markwell J: Assays for determination of protein concentration. Curr Protoc Protein Sci Chapter 3: Unit 3.4, 2007.

28. Tsuchiya M, Takaoka A, Tokioka N and Matsuura S: Development of an endotoxin-specific Limulus amebocyte lysate test blocking beta-glucan-mediated pathway by carboxymethylated curdlan and its application. Nihon Saikingaku Zasshi 45: 903-911, 1990 (In Japanese).

29. Malich G, Markovic B and Winder C: The sensitivity and specificity of the MTS tetrazolium assay for detecting the in vitro cytotoxicity of 20 chemicals using human cell lines. Toxicology 124: 179-192, 1997.

30. Croucher PI, Shipman CM, Lippitt J, et al: Osteoprotegerin inhibits the development of osteolytic bone disease in multiple myeloma. Blood 98: 3534-3540, 2001.

31. Burgess-Brown NA, Sharma S, Sobott F, Loenarz C, Oppermann U and Gileadi O: Codon optimization can improve expression of human genes in Escherichia coli: A multi-gene study. Protein Expr Purif 59: 94-102, 2008. 
32. Wu X, Jornvall H, Berndt KD and Oppermann U: Codon optimization reveals critical factors for high level expression of two rare codon genes in Escherichia coli: RNA stability and secondary structure but not tRNA abundance. Biochem Biophys Res Commun 313: 89-96, 2004.

33. Kreitman RJ and Pastan I: Immunobiological treatments of hairy-cell leukaemia. Best Pract Res Clin Haematol 16: 117-133, 2003.

34. Pastan I, Hassan R, Fitzgerald DJ and Kreitman RJ: Immunotoxin therapy of cancer. Nat Rev Cancer 6: 559-565, 2006.

35. Pastan I, Beers R and Bera TK: Recombinant immunotoxins in the treatment of cancer. Methods Mol Biol 248: 503-518, 2004.

36. Chiron MF, Fryling CM and FitzGerald D: Furin-mediated cleavage of Pseudomonas exotoxin-derived chimeric toxins. J Biol Chem 272: 31707-31711, 1997.

37. Bassi DE, Lopez De Cicco R, Mahloogi H, Zucker S, Thomas G and Klein-Szanto AJ: Furin inhibition results in absent or decreased invasiveness and tumorigenicity of human cancer cells. Proc Natl Acad Sci USA 98: 10326-10331, 2001.

38. Thomas G: Furin at the cutting edge: from protein traffic to embryogenesis and disease. Nat Rev Mol Cell Biol 3: 753-766, 2002.

39. Bassi DE, Fu J, Lopez de Cicco R and Klein-Szanto AJ: Proprotein convertases: 'master switches' in the regulation of tumor growth and progression. Mol Carcinog 44: 151-161, 2005.

40. Alberti L, Bachelot T, Duc A, Biota C and Blay JY: A spliced isoform of interleukin 6 mRNA produced by renal cell carcinoma encodes for an interleukin 6 inhibitor. Cancer Res 65: 2-5, 2005.

41. Scheller J and Rose-John S: Interleukin-6 and its receptor: from bench to bedside. Med Microbiol Immunol 195: 173-183, 2006.

42. Wong VW, Yu J, Cheng AS, et al: High serum interleukin-6 leve predicts future hepatocellular carcinoma development in patients with chronic hepatitis B. Int J Cancer 124: 2766-2770, 2009.
43. Hodge DR, Hurt EM and Farrar WL: The role of IL-6 and STAT3 in inflammation and cancer. Eur J Cancer 41: 2502-2512, 2005.

44. Song S, Xue J, Fan K, et al: Preparation and characterization of fusion protein truncated Pseudomonas Exotoxin A (PE38KDEL) in Escherichia coli. Protein Expr Purif 44: 52-57, 2005

45. Nesbitt JE and Fuller GM: Dynamics of interleukin-6 internalization and degradation in rat hepatocytes. J Biol Chem 267: $5739-5742,1992$

46. Lorberboum-Galski H, Garsia RJ, Gately M, et al: IL2PE664Glu, a new chimeric protein cytotoxic to human-activated T lymphocytes. J Biol Chem 265: 16311-16317, 1990.

47. Mori K, Fujimoto-Ouchi K, Onuma E, et al: Novel models of cancer-related anemia in mice inoculated with IL-6-producing tumor cells. Biomed Res 30: 47-51, 2009.

48. Jongen-Lavrencic M, Peeters HR, Rozemuller H, et al: IL-6induced anaemia in rats: possible pathogenetic implications for anemia observed in chronic inflammations. Clin Exp Immunol 103: 328-334, 1996.

49. Raj DS: Role of interleukin-6 in the anemia of chronic disease. Semin Arthritis Rheum 38: 382-388, 2009.

50. Wierzbowska A, Urbanska-Rys H and Robak T: Circulating IL-6-type cytokines and sIL-6R in patients with multiple myeloma. Br J Haematol 105: 412-419, 1999.

51. Usnarska-Zubkiewicz L: Level of interleukin-6 (IL-6), soluble interleukin-6 receptors (sIL-6R) and tumor necrosis factor alpha (TNF-alpha) in untreated and progressing multiple myeloma. Pol Arch Med Wewn 99: 30-37, 1998 (In Polish).

52. Tsutsumi Y, Onda M, Nagata S, Lee B, Kreitman RJ and Pastan I: Site-specific chemical modification with polyethylene glycol of recombinant immunotoxin anti-Tac(Fv)-PE38 (LMB-2) improves antitumor activity and reduces animal toxicity and immunogenicity. Proc Natl Acad Sci USA 97: 8548-8553, 2000. 